\title{
Red giants in open clusters ${ }^{\star}$
}

\section{NGC 1817}

\author{
J.-C. Mermilliod ${ }^{1}$, D. W. Latham ${ }^{2}$, E. V. Glushkova ${ }^{3}$, M. A. Ibrahimov ${ }^{4}$, V. M. Batirshinova ${ }^{4}$, \\ R. P. Stefanik ${ }^{2}$, and D. J. James ${ }^{1,5}$ \\ 1 Institut d'Astronomie de l'Université de Lausanne, 1290 Chavannes-des-Bois, Switzerland \\ 2 Harvard-Smithsonian Center for Astrophysics, 60 Graden Street, Cambridge, Massachusetts 02138, USA \\ 3 Sternberg Astronomical Institute, Universitetskii pr. 13, Moscow 119899, Russia \\ ${ }^{4}$ Ulugh Beg Astronomical Institute, Academy of Sciences of Uzbekistan, Astronomicheskaya str. 33, Tashkent 700052, \\ Uzbekistan \\ 5 School of physics and Astronomy, University of St Andrews, St Andrews, Fife, KY169SS, UK
}

Received 13 June 2002 / Accepted 12 November 2002

\begin{abstract}
Radial-velocity and $B V$ CCD observations of 29 red-giant candidates in the central part of the intermediate-age open cluster NGC 1817 have confirmed the membership of 21 stars. In addition, we have used radial velocities and new $U B V R$ photoelectric photometry to investigate the membership of 59 new candidates located in an outer corona surrounding NGC 1817, identified from the POSS plates. We have confirmed 18 new members out to a distance of $27^{\prime}$ from the centre, thus doubling the previous cluster radius. Ten definite spectroscopic-binary members have been discovered, corresponding to a binary frequency of $25.6 \%$ and four orbits have been determined so far. Three additional stars have been identified as possible spectroscopicbinary members. We derive a cluster mean velocity of $+65.33 \pm 0.09(0.52 \mathrm{s.e}$. $) \mathrm{km} \mathrm{s}^{-1}$ based on 29 members with no detected velocity variations plus the 3 binary members with orbital solutions. The radial distribution of the 39 red-giant members shows a strong concentration of spectroscopic binaries in the inner part of the cluster, similar to that observed in M 67, although NGC 1817 is much younger.
\end{abstract}

Key words. open clusters and associations: individual: NGC 1817 - stars: binaries: spectroscopic - techniques: radial velocities - stars: AGB and post-AGB

\section{Introduction}

The physical limits of open clusters are difficult to define. The apparent diameters tabulated mainly by Lyngå (1987) are based on eye estimates, which depend on the density ratio of the cluster to the background field. In many cases, the true cluster extension can be significantly larger than the area investigated, and this has several important consequences for the determination of the luminosity function, the study of the radial structure, and on the distribution of stellar populations in the

Send offprint requests to: J.-C. Mermilliod, e-mail: Jean-Claude.Mermilliod@obs.unige.ch

* Based on observations collected at the Haute-Provence Observatory (France), and with the Jacobus Kapteyn Telescope, operated on the island of La Palma by PPARC at the Spanish Observatorio del Roque de los Muchachos. Some of the radialvelocity observations were obtained with the Multiple Mirror Telescope, a joint facility of the Smithsonian Institution and the University of Arizona. colour-magnitude diagram. The latter point is especially important for the study of red-giant evolution.

The photometric investigation of NGC 1817 by Harris \& Harris (1977) led us to believe that the area they studied was too small with respect to the real size of the cluster. Therefore the POSS charts were examined to identify red stars in the area surrounding NGC 1817 out to $45^{\prime}$ from the centre, while the radius given by Lyngå (1987) is $10^{\prime}$. Fifty-nine stars were selected for further observations. We show in this paper that 18 of the 59 outer candidates have radial velocities and $U B V R$ photometry consistent with cluster membership.

The total number of red giants in NGC 1817 is now 39 (21 in the inner part and 18 in the outer part). Thus, this cluster becomes very interesting for comparing the radial distributions of the single and binary red giants, to extend the work of Raboud \& Mermilliod (1994). It also provides another cluster for comparing the observed patterns of red giants in the colour-magnitude diagrams with those predicted by theoretical isochrones, in connection with the apparent mass spread among 
clump red giants discovered by Girardi et al. (2000) and the peculiar structure of the clump it produces.

The intermediate-age open cluster NGC 1817 (C0509+166, $\left.\operatorname{RA}(2000)=5 \mathrm{~h} 12^{\mathrm{m}} 1, \operatorname{Dec}(2000)=+16^{\circ} 42^{\prime}\right)$ has been observed in $U B V$ photometry, both photoelectric and photographic, by Purgathofer (1964) and Harris \& Harris (1977). Its distance is close to $2 \mathrm{kpc}$, the reddening is $E(B-V)=0.33$, and the age is $\log t=8.75$. Proper motions and membership probabilities have been published by Tian et al. (1983) and Balaguer-Núñez et al. (1998). Friel \& Janes (1993) obtained radial velocities for five stars and derived a value for $[\mathrm{Fe} / \mathrm{H}]$ equal to -0.39 . The numbering system adopted for this cluster is based on the study of Cuffey (1938). Paper IX in this series was devoted to four open clusters (Mermilliod et al. 2001) where further references to previous papers can be found.

In Sect. 2 we describe the sample and the observations. We present the results in Sect. 3 and the discussions on the colourmagnitude diagram and mass segregation in Sects. 4 and 5 respectively.

\section{Observations}

\subsection{The sample}

An initial inner sample of 23 red-giant candidates was selected from the $U B V$ study of Harris \& Harris (1977). An additional 6 inner stars, C121, C185, C187, C211, C212, C265 and $\mathrm{C} 267$, were selected on the basis of their magnitudes and colours from Cuffey (1938). It is obvious from the POSS image of the cluster that the field investigated by Harris \& Harris (1977) is too small. Therefore, candidate red giants were identified in an outer corona surrounding the cluster core by (EVG) on both $\mathrm{O}$ and $\mathrm{E}$ glass copies of the POSS atlas with a blink comparator. 434 red stars were selected in this way. Then photometry was made on the same plates and $\mathrm{O}$ and $\mathrm{E}$ magnitudes were converted to $V$ and $B$. The final selection yielded a list of 59 outer red-giant candidates. Radial velocities and photoelectric $U B V R$ photometry were obtained for these stars to investigate their membership.

\subsection{Radial-velocity observations}

\subsubsection{First campaign}

Observations were obtained with the CORAVEL radialvelocity scanner (Baranne et al. 1979) installed on the Danish 1.54-m telescope at La Silla (ESO, Chile) over several seasons. For candidates in the inner part of the cluster at least three observations were obtained for member stars, and usually two for non-members. The first observations were obtained in 1986, but the bulk of the radial velocities were obtained between 1993 and 1996.

Candidates in the outer part of the cluster were observed in order of increasing distance from the centre to build a radially complete sample. Stars that could not be observed due to lack of telescope time are located in the outermost part of the corona. None of the stars observed in the outermost ring appear to be members, so the number of members missed should be small.
Ten definite spectroscopic-binary members were discovered, and two bright ones, C44 and C164, were also observed from the Haute-Provence Observatory (France) with the CORAVEL installed on the Swiss 1-m telescope. Radialvelocity observations of several binaries were also obtained by (RPS) with the Harvard-Smithsonian Center for Astrophysics (CfA) Digital Speedometers (Latham 1992), especially to improve the phase coverage for stars C44 and C164.

\subsubsection{Second campaign}

A second campaign of observations was undertaken in the season 2000-2001 to address the referee's criticism that the binarity of the red giants in the outer part of the cluster was not well established. Because the CORAVELs are no longer in operation, the new radial velocities were obtained by (DWL and RPS) with the CfA Digital Speedometers (Latham 1992). The errors for a single observation are of the order of $0.5 \mathrm{~km} \mathrm{~s}^{-1}$.

One additional measurement was obtained for most of the inner red giants, a second observation was obtained for the outer red giants already observed with CORAVEL, and all stars not observed with CORAVEL were measured at least once at CfA. As a consequence, all the stars in the initial sample now have radial-velocity observations. In addition, several new observations were obtained for the spectroscopic binaries, allowing two additional orbits to be solved, for star C56 and \#1081 (T81).

The individual observations (Table 1) are available in electronic form from the Strasbourg service for published tables (http://cdsweb.u-strasbg.fr/cgi-bin/qcat? J/A+A/399/105). Only the beginning is shown here to illustrate the Table format, which contains the star identification, the telescope $(\mathrm{C}=$ CORAVEL, $\mathrm{W}=$ Wyeth Reflector, $\mathrm{T}=$ Tillinghast Reflector, $\mathrm{M}=\mathrm{MMT}$ ) the Julian Date, and the radial velocity and internal error estimate, in $\mathrm{km} \mathrm{s}^{-1}$.

The CORAVEL radial velocities are on the system defined by Udry et al. (1999) based on high-precision radial velocities obtained with the ELODIE spectrograph (Baranne et al. 1996). This calibration corrects for the known systematic errors of the CORAVEL systems. The CfA radial velocities are on an absolute system defined by extensive observations of minor planets, as described by Stefanik et al. (1999), except that $0.139 \mathrm{~km} \mathrm{~s}^{-1}$ have been added to the velocities on the native CfA system to put them on an absolute system, rather than subtracted, as was specified in error by Stefanik et al. (1999). To test the compatibility of the zero points of the CORAVEL and CfA velocity systems, we calculated the mean velocity difference using 28 members observed with both systems and no sign of velocity variation. The mean difference CfA-CORAVEL was $-0.001 \pm 0.105 \mathrm{~km} \mathrm{~s}^{-1}$, thus confirming that the two data sets could be merged without any adjustment of the velocity zero points.

Anticipating on the following discussion, it is worth noting that the new observations beautifully confirm the previous results, both for the membership and binarity. 
Table 1. Sample of individual radial velocities in NGC 1817. Full table available at the CDS via anonymous ftp to cdsarc.u-strasbg.fr $(130.79 .128 .5)$ or via http://cdsweb.u-strasbg.fr/cgi-bin/qcat?]/A+A/399/105.

\begin{tabular}{rrccc}
\hline \hline No. & Tel & JD & $V_{\mathrm{r}}$ & $\sigma$ \\
\hline 0008 & $\mathrm{C}$ & 2449373.546 & 64.35 & 0.62 \\
0008 & $\mathrm{C}$ & 2450082.611 & 65.15 & 0.39 \\
0008 & $\mathrm{~T}$ & 2451833.985 & 65.20 & 0.40 \\
0008 & $\mathrm{~T}$ & 2451919.831 & 65.25 & 0.45 \\
0012 & $\mathrm{C}$ & 2446466.582 & 61.95 & 0.82 \\
\hline
\end{tabular}

\subsection{Photoelectric UBVR observations}

Photoelectric $U B V R$ observations of the candidate red giants in the cluster corona were made during 1997-98 with a singlechannel pulse-counting photometer installed on the 0.6-m Zeiss telescope at Mt. Maidanak High-altitude Observatory of the Ulugh Beg Astronomical Institute (Uzbekistan). BD $+8^{\circ} 1051$ was used as the comparison star; SA 96-36 and SA 97-351 as the check stars. Their magnitudes were taken from Landolt (1992). The mean precision of the $V$-magnitude measurements is $0.015 \mathrm{mag}$, whereas for the colours $(B-V),(U-B),(V-R)$, it amounts to $0.02,0.05$ and $0.02 \mathrm{mag}$, respectively. The results of the $U B V R$ observations are listed in Table 3.

\section{4. $C C D B V$ observations}

Only photographic photometry was available for many of the red giants in the cluster core. To analyse the structure of the clump accurate data are needed, and we decided to obtain CCD data for that purpose. CCD $B V$ observations were taken during the night of November 2, 1999 by (DJJ). A Harris filter set and a SITe $2048 \times 2048$ CCD chip were used together the 1.0-m Jacobus Kapteyn Telescope, located at the Observatorio del Roque de Los Muchachos on La Palma, in the Canary Islands. Standard star exposures were taken throughout the night at a variety of airmasses, using stars in the Standard Area fields 95, 98 and 111 from Landolt (1992). The mean internal precision of the standard $V$-magnitude measurements is 0.026 , while for the $B-V$ measurements it is 0.040 . These errors are most likely dominated by minor flat-fielding problems during data-reduction, and the relative dimness of the standard stars used. The $V$ and $B-V$ magnitudes of the target stars are detailed in Table 2.

\section{Results}

Results for the radial-velocity and CCD $B V$ observations of the inner red giants are given in Table 2 . The columns contain successively the Cuffey (1938) and Harris \& Harris (1977) numbers, the mean radial velocities and errors $\left(\mathrm{km} \mathrm{s}^{-1}\right)$, the number of observations, the time interval spanned by the observations, the probability $P\left(\chi^{2}\right)$ that the scatter is only due to random noise, and remarks on duplicity and membership (SB: spectroscopic binary, SBO: single-lined orbit determined, and NM: non-member).
Table 2. CCD $B V$ and radial-velocity results for the inner red giants.

\begin{tabular}{|c|c|c|c|c|c|c|c|c|c|}
\hline No. & $\mathrm{HH}$ & $V$ & $B-V$ & $V_{\mathrm{r}}$ & $\epsilon$ & $n$ & $\Delta T$ & $P\left(\chi^{2}\right)$ & Rem \\
\hline 8 & 1027 & 12.13 & 1.03 & +64.99 & 0.27 & 4 & 2546 & 0.603 & \\
\hline 12 & 1014 & 12.58 & 0.98 & +67.82 & 0.93 & 12 & 5448 & 0.000 & SB \\
\hline 19 & 2112 & 12.22 & 1.04 & +62.45 & 2.36 & 8 & 5444 & 0.000 & SB \\
\hline 22 & 2104 & 12.37 & 1.06 & +64.79 & 0.54 & 8 & 5444 & 0.000 & SB \\
\hline 30 & 1061 & 12.43 & 1.07 & +64.79 & 0.49 & 4 & 5444 & 0.265 & \\
\hline 40 & 1051 & 12.68 & 1.07 & +65.86 & 0.41 & 4 & 5444 & 0.143 & \\
\hline 44 & 1044 & & & +65.69 & 0.16 & 24 & 5448 & 0.000 & SBO \\
\hline 56 & 3134 & 11.22 & 1.40 & +65.00 & 0.12 & 18 & 5539 & 0.000 & SBO \\
\hline 64 & 3114 & 12.16 & 1.09 & +65.14 & 0.31 & 4 & 2896 & 0.510 & \\
\hline 71 & 2063 & 12.81 & 1.05 & +65.88 & 0.37 & 4 & 2896 & 0.795 & \\
\hline 72 & 2060 & 12.03 & 1.18 & +63.74 & 0.56 & 5 & 2896 & 0.000 & SB \\
\hline 73 & 2059 & 12.04 & 1.08 & +65.52 & 0.41 & 4 & 2896 & 0.137 & \\
\hline 79 & 1038 & 12.46 & 1.08 & +64.96 & 0.40 & 4 & 2815 & 0.279 & \\
\hline 81 & 2038 & & & +64.92 & 0.28 & 5 & 2901 & 0.804 & \\
\hline 121 & & 12.73 & 1.04 & +64.61 & 0.35 & 4 & 2542 & 0.874 & \\
\hline 127 & 3106 & 12.27 & 1.09 & +65.31 & 0.22 & 4 & 2896 & 0.932 & \\
\hline 164 & 2042 & 11.73 & 0.81 & +64.69 & 0.14 & 26 & 5095 & 0.000 & SBO \\
\hline 177 & 2009 & 12.31 & 1.07 & +65.18 & 0.33 & 4 & 2814 & 0.754 & \\
\hline 211 & & 12.30 & 1.07 & +64.99 & 0.49 & 4 & 2895 & 0.087 & \\
\hline 212 & & 12.04 & 1.41 & +65.81 & 0.33 & 4 & 2895 & 0.973 & \\
\hline 244 & 3031 & 12.58 & 1.05 & +64.54 & 0.99 & 10 & 2895 & 0.000 & SB \\
\hline 90 & 1008 & & & +29.75 & 0.50 & 3 & 2949 & 0.100 & NM \\
\hline 103 & 2124 & & & -9.95 & 0.43 & 2 & 356 & 0.221 & NM \\
\hline 138 & 3094 & & & +8.12 & 0.30 & 2 & 354 & 0.371 & NM \\
\hline 155 & 3066 & & & +14.40 & 0.15 & 3 & 2974 & 0.779 & NM \\
\hline 161 & 2047 & & & -3.08 & 0.35 & 3 & 4794 & 0.174 & NM \\
\hline 187 & & & & +19.89 & 0.12 & 4 & 3339 & 0.872 & NM \\
\hline 265 & & & & +71.71 & 0.36 & 3 & 744 & 0.286 & NM \\
\hline 269 & & & & -21.78 & 0.33 & 2 & 355 & 0.752 & NM \\
\hline
\end{tabular}

Observations for the corona candidates are summarized in Table 3 which contains: the star identifications adopted in the open cluster database (WEBDA: http://obswww.unige.ch/webda/): numbers larger than 1000 come from the proper-motion study of Tian et al. (1983), the current number from this study (EG) and that used by Balaguer-Núñez et al. (1998) (Bal). The coordinates for Epoch 2000 and membership probabilities (P) are also from Balaguer-Núñez et al. (1998). Next, five columns detail the $U B V R$ photometric results, and the five following represent the radial-velocity data in a form similar to that of Table 2. The last column shows remarks on membership (M?: doubtful member) and duplicity (SB: spectroscopic binary).

\subsection{Membership}

\subsubsection{Inner stars}

The membership of the red-giant candidates in the inner part of NGC 1817 is easy to determine. There are enough observations to compute reliable mean velocities, and the non-members stand out unambiguously, with radial velocity differences from the cluster mean of more than $30 \mathrm{~km} \mathrm{~s}^{-1}$, except for star C265, which differs by $6 \mathrm{~km} \mathrm{~s}^{-1}$. The 14 most central stars are members, but only half of the candidates are confirmed as members for distances from the centre in the range $6^{\prime}<R<10^{\prime}$. 
Table 3. Photoelectric $U B V R$ and radial-velocity results for the outer red giants.

\begin{tabular}{|c|c|c|c|c|c|c|c|c|c|c|c|c|c|c|c|c|}
\hline \multirow[t]{2}{*}{ No. } & \multirow[t]{2}{*}{ EG } & \multirow[t]{2}{*}{ Bal } & \multicolumn{2}{|c|}{ RA (2000) Dec } & $P$ & $V$ & $U-B$ & $B-V$ & $V-R$ & $N$ & $V_{\mathrm{r}}$ & $\epsilon$ & $n$ & $\Delta T$ & $P\left(\chi^{2}\right)$ & Rem \\
\hline & & & \multicolumn{14}{|c|}{ Members } \\
\hline 185 & 30 & 395 & 51231.00 & +164925.4 & .99 & 12.85 & 0.72 & 1.12 & 0.91 & 1 & +64.99 & 0.40 & 3 & 2542 & 0.736 & \\
\hline 206 & 11 & 343 & 51301.80 & +164114.4 & .98 & 11.92 & 0.96 & 1.26 & 1.02 & 2 & +65.46 & 0.41 & 3 & 2154 & 0.141 & \\
\hline 673 & 3 & & 51221.10 & +162650.9 & & 12.68 & 0.69 & 1.03 & 0.86 & 1 & +65.77 & 0.35 & 3 & 2157 & 0.336 & \\
\hline 677 & 20 & & 51253.66 & +164933.9 & & 12.61 & 0.77 & 1.12 & 0.95 & 1 & +65.30 & 0.40 & 3 & 2159 & 0.274 & \\
\hline 1114 & 48 & 735 & 51127.55 & +164704.6 & .98 & 12.82 & 0.72 & 1.11 & 0.95 & 2 & +65.72 & 0.36 & 3 & 2159 & 0.512 & \\
\hline 1117 & 42 & 600 & 51149.93 & +165042.0 & .98 & 13.33 & 0.14 & 0.61 & 0.61 & 1 & +66.22 & 0.50 & 3 & 2160 & 0.066 & \\
\hline 1135 & 47 & 714 & 51139.32 & +164009.3 & .99 & 12.51 & 0.66 & 1.11 & 0.93 & 2 & +64.56 & 0.66 & 3 & 2071 & 0.036 & \\
\hline 1152 & 54 & 816 & 51048.19 & +162840.1 & .24 & 12.16 & 0.74 & 1.14 & 0.94 & 2 & +65.81 & 0.53 & 2 & 1748 & 0.897 & \\
\hline 1208 & 57 & 689 & 51110.74 & +162641.3 & .32 & 12.35 & 0.71 & 1.11 & 0.89 & 2 & +64.77 & 5.28 & 8 & 2155 & 0.000 & SB \\
\hline 1265 & 34 & 463 & 51252.13 & +170816.4 & .95 & 12.50 & 0.64 & 1.04 & 0.89 & 1 & +65.66 & 0.50 & 1 & & & \\
\hline 1292 & 31 & 605 & 51219.67 & +165423.2 & .99 & 12.29 & 0.70 & 1.11 & 0.93 & 1 & +65.72 & 0.32 & 3 & 2102 & 0.868 & \\
\hline 1408 & 8 & 301 & 51306.24 & +163114.2 & .95 & 12.81 & 0.73 & 1.08 & 0.93 & 2 & +65.98 & 0.33 & 3 & 2158 & 0.850 & \\
\hline 1412 & 7 & 150 & 51313.83 & +163324.4 & .94 & 12.69 & 0.75 & 1.13 & 0.97 & 2 & +67.03 & 0.34 & 3 & 2158 & 0.719 & \\
\hline 1420 & 13 & 160 & 51339.67 & +163916.3 & .79 & 12.62 & 1.01 & 0.54 & 0.88 & 1 & +59.49 & 3.75 & 4 & 2190 & 0.000 & SB \\
\hline 1433 & 15 & 180 & 51341.76 & +164329.0 & .81 & 12.24 & 0.70 & 1.08 & 0.93 & 1 & +65.46 & 0.35 & 3 & 2155 & 0.387 & \\
\hline 1456 & 1 & 292 & 51232.83 & +162825.3 & .98 & 11.43 & 1.10 & 1.26 & 0.98 & 1 & +66.04 & 0.98 & 4 & 2191 & 0.000 & SB \\
\hline 1459 & 9 & 296 & 51250.89 & +162857.8 & .96 & 11.93 & 0.81 & 1.13 & 0.88 & 1 & +66.06 & 0.33 & 3 & 2157 & 0.944 & \\
\hline \multirow[t]{2}{*}{1574} & 18 & 65 & 51412.58 & +164145.1 & .18 & 12.17 & 0.67 & 1.13 & 0.92 & 1 & +65.99 & 0.48 & 2 & 1836 & 0.333 & \\
\hline & & & \multicolumn{14}{|c|}{ Non members } \\
\hline 531 & 23 & 432 & 51259.42 & +165948.0 & .97 & 12.48 & 0.60 & 1.01 & 0.88 & 1 & -20.70 & 0.52 & 1 & & & \\
\hline 536 & 24 & 448 & 51302.07 & +170302.7 & .87 & 12.41 & 0.81 & 1.18 & 0.95 & 1 & +7.05 & 0.70 & 1 & & & \\
\hline 571 & 32 & 610 & 51201.39 & +165643.1 & .93 & 12.86 & 1.28 & 1.55 & 1.26 & 1 & +26.01 & 0.64 & 1 & & & \\
\hline 598 & 53 & 696 & 51121.84 & +163148.5 & .43 & 11.87 & 0.65 & 1.11 & 0.93 & 2 & +74.51 & 0.57 & 2 & 321 & 0.862 & \\
\hline 621 & 43 & 770 & 51128.83 & +170332.9 & .01 & 11.57 & 0.95 & 1.23 & 1.00 & 1 & -22.98 & 1.12 & 2 & 264 & 0.021 & \\
\hline 674 & 5 & & 51211.56 & +160612.3 & & 11.87 & 1.23 & 1.29 & 1.01 & 1 & +76.07 & 0.52 & 2 & 85 & 0.301 & \\
\hline 675 & 17 & & 51447.71 & +164506.7 & & 11.85 & 0.97 & 1.20 & 0.98 & 1 & -13.14 & 0.47 & 1 & & & \\
\hline 676 & 19 & & 51445.68 & +162933.5 & & 12.87 & 1.34 & 1.33 & 1.10 & 1 & +9.39 & 0.47 & 1 & & & \\
\hline 678 & 29 & & 51443.09 & +165400.9 & & 12.63 & 0.76 & 1.09 & 0.95 & 1 & +32.57 & 0.58 & 1 & & & \\
\hline 679 & 37 & & 51229.40 & +171159.2 & & 13.01 & 0.07 & 0.70 & 0.59 & 1 & +36.19 & 0.63 & 1 & & & \\
\hline 680 & 40 & & 51145.00 & +17 1221.2 & & 11.75 & 1.08 & 1.23 & 0.97 & 1 & +2.79 & 0.38 & 3 & 134 & 0.484 & \\
\hline 681 & 41 & & 51138.93 & +171342.2 & & 12.35 & 0.89 & 1.13 & 0.96 & 1 & +25.60 & 0.50 & 1 & & & \\
\hline 682 & 52 & & 51009.00 & +163424.9 & & 12.32 & 0.71 & 1.15 & 0.96 & 2 & +111.53 & 0.40 & 2 & 29 & 0.440 & \\
\hline 1081 & 46 & 885 & 51050.99 & +165337.4 & .00 & 11.45 & 0.12 & 0.73 & 0.71 & 1 & +38.81 & 6.88 & 12 & 464 & 0.000 & SB \\
\hline 1082 & 45 & 886 & 51054.43 & +165353.0 & .92 & 12.24 & 0.73 & 1.13 & 0.93 & 1 & +33.65 & 0.53 & 1 & & & \\
\hline 1083 & 44 & 896 & 51055.48 & +165810.8 & .95 & 11.86 & 1.66 & 1.63 & 1.34 & 1 & +41.59 & 0.45 & 1 & & & \\
\hline 1095 & 51 & 870 & 51042.54 & +164610.0 & .93 & 12.22 & 1.58 & 1.55 & 1.25 & 2 & +12.20 & 0.42 & 1 & & & \\
\hline 1096 & 50 & 863 & 51046.84 & +164438.5 & .95 & 12.71 & 1.57 & 1.48 & 1.20 & 2 & -41.77 & 0.49 & 1 & & & \\
\hline 1112 & 49 & 733 & 51115.60 & +164623.5 & .98 & 12.78 & 0.74 & 1.13 & 0.98 & 2 & +3.19 & 0.47 & 1 & & & \\
\hline 1153 & 55 & 815 & 51046.92 & +162820.6 & .48 & 11.82 & 1.54 & 1.46 & 1.16 & 2 & +28.49 & 0.49 & 1 & & & \\
\hline 1161 & 56 & 811 & 51023.16 & +162718.3 & .09 & 11.84 & 0.89 & 1.15 & 0.93 & 2 & +11.71 & 0.49 & 1 & & & \\
\hline 1194 & 58 & 674 & 51127.84 & +161705.2 & .01 & 12.10 & 1.23 & 1.35 & 1.09 & 2 & +50.85 & 0.40 & 1 & & & \\
\hline 1197 & 59 & 672 & 51104.11 & +161702.7 & .05 & 11.27 & 1.10 & 1.27 & 1.00 & 2 & +56.17 & 0.33 & 4 & 2155 & 0.436 & \\
\hline 1246 & 36 & 468 & 51236.31 & +171136.6 & .02 & 12.97 & 0.24 & 0.61 & 0.87 & 1 & +4.76 & 0.68 & 1 & & & \\
\hline 1248 & 35 & 467 & 51241.11 & +171102.1 & .91 & 11.82 & 0.50 & 1.00 & 0.85 & 2 & +19.08 & 0.50 & 1 & & & \\
\hline 1252 & 38 & 660 & 51222.50 & +170929.0 & .00 & 12.54 & 0.79 & 1.21 & 0.98 & 1 & -27.96 & 0.50 & 1 & & & \\
\hline 1254 & 39 & 648 & 51159.70 & +170542.8 & .95 & 12.16 & 1.01 & 1.47 & 0.84 & 1 & +60.18 & 0.30 & 4 & 2097 & 0.915 & \\
\hline 1267 & 25 & 237 & 51325.29 & +170920.8 & .00 & 11.71 & 1.26 & 1.19 & 1.03 & 1 & -20.49 & 0.60 & 2 & 47 & 0.730 & \\
\hline 1273 & 26 & 112 & 51359.10 & +170804.4 & .00 & 12.78 & 0.91 & 1.21 & 1.03 & 1 & -10.26 & 0.47 & 1 & & & \\
\hline 1297 & 33 & 430 & 51247.99 & +165920.7 & .98 & 12.64 & 0.62 & 1.05 & 0.88 & 1 & +60.00 & 0.37 & 3 & 2157 & 0.741 & \\
\hline 1302 & 22 & 423 & 51303.59 & +165804.3 & .98 & 11.31 & 0.73 & 1.10 & 0.89 & 1 & +38.49 & 0.37 & 2 & 2202 & 0.618 & \\
\hline 1314 & 21 & 206 & 51322.43 & +165217.4 & .88 & 12.06 & 0.70 & 1.07 & 0.88 & 1 & -2.96 & 0.46 & 1 & & & \\
\hline 1316 & 27 & 214 & 51340.46 & +165430.2 & .89 & 11.70 & 1.77 & 1.54 & 1.23 & 1 & -6.05 & 0.50 & 2 & 2198 & 0.720 & \\
\hline 1406 & 6 & 304 & 51254.20 & +163247.8 & .98 & 12.55 & 0.88 & 1.12 & 0.97 & 1 & +24.73 & 0.50 & 1 & & & \\
\hline 1418 & 16 & 56 & 51403.84 & +163751.3 & .02 & 11.73 & 0.90 & 1.18 & 0.97 & 1 & +14.43 & 0.75 & 2 & 2201 & 0.156 & \\
\hline 1424 & 12 & 3 & 51309.76 & +164142.9 & .96 & 12.09 & 1.06 & 1.21 & 0.99 & 2 & +50.78 & 0.45 & 2 & 322 & 0.550 & \\
\hline 1455 & 2 & 502 & 51223.66 & +162811.3 & .98 & 12.68 & 0.91 & 1.08 & 0.88 & 1 & +7.23 & 0.48 & 1 & & & \\
\hline 1467 & 4 & 493 & 51206.32 & +162104.1 & .00 & 12.49 & 0.46 & 0.91 & 0.84 & 1 & -5.82 & 0.72 & 1 & & & \\
\hline 1502 & 10 & 122 & 51332.54 & +161714.0 & .00 & 12.80 & 1.03 & 1.21 & 1.01 & 1 & +60.94 & 0.51 & 1 & & & \\
\hline 1557 & 28 & 91 & 51429.25 & +165241.6 & .00 & 12.42 & 1.63 & 1.48 & 1.20 & 1 & +33.52 & 0.50 & 1 & & & \\
\hline 1718 & 14 & 162 & 51339.86 & +163927.3 & .60 & 13.70 & 0.07 & 0.73 & 0.58 & 1 & +62.08 & 1.62 & 2 & 2195 & 0.022 & SB? \\
\hline
\end{tabular}


Star C72 is now identified as a binary with a long period. Star C90 observed by Friel \& Janes (1993) is a non-member according to our observations.

\subsubsection{Outer stars}

The radial-velocity observations of the 59 candidates in the outer region of the cluster now confirm the membership of 18 red giants. Most of them also have high membership probabilities from the proper-motion study of Balaguer-Núñez et al. (1998). The new data confirm all the memberships deduced from the first observing campaign, and permit the tentative confirmation of an additional member, star \#1265 (Tian 265), based on a single velocity observation. The mean radial velocities of the members range from +64.51 to $67.03 \mathrm{~km} \mathrm{~s}^{-1}$, i.e. $2.5 \mathrm{~km} \mathrm{~s}^{-1}$.

Star 1297 (EG33) has been rejected from membership because its mean velocity, based on 3 observations taken over 2157 days, is stable and off by $5 \mathrm{~km} \mathrm{~s}^{-1}$ (i.e. $10 \sigma$ ) from the cluster mean. The membership of star \#1718 (Tian 718) is important in view of our interest to mass segregation. We have a single CORAVEL velocity of $60.93 \pm 0.67 \mathrm{~km} \mathrm{~s}^{-1}$ followed by a single CfA velocity six years later at $63.24 \pm 0.76 \mathrm{~km} \mathrm{~s}^{-1}$. This star is probably a spectroscopic binary, it however cannot be a member. Its colour indices indicate that \#1718 is a dwarf are therefore inconsistent with membership. With $V=$ 13.70, $B-V=0.73$, star \#1718 does not appear in Fig. 4. The $V$ magnitude and $B-V$ colour have been confirmed by independant observations obtained by Leonid Berdnikov. All the other outer candidates are probably non-members with $\left(\Delta V_{\mathrm{r}}>10 \sigma=5.20 \mathrm{~km} \mathrm{~s}^{-1}\right)$.

For stars with only one radial-velocity observation our identification of non-members is unambiguous only if the stars are not binaries. All of the radial-velocity non-members are located away from the cluster locus in the colour-magnitude diagram, thus supporting their non-member classification. Although the proper-motion memberships are not very decisive for this cluster, they often support our non-member identifications. The membership or non-membership of C1502 $\left(V_{\mathrm{r}}=\right.$ $\left.+60.74 \mathrm{~km} \mathrm{~s}^{-1} n=1\right)$ is still ambiguous and awaits additional observations. If a member, this star should be a binary. It is also located away from the cluster locus in the colour-magnitude diagram.

\subsection{Binarity}

Ten definite and one probable (\#1456) spectroscopic-binary members were discovered and four orbits were determined, for three members, C44, C56, C164 and one nonmember, \#1081. The latter is based on 12 observations and therefore preliminary. Orbital elements are given in Table 4 and radial-velocity curves are shown in Figs. 1, 2 and 3. The eccentricity of the orbit for $\mathrm{C} 44$ is indistinguishable from zero, which is expected because the period is shorter than the cut-off period for circular orbits at 120-150 days (Mermilliod \& Mayor 1996). For C164 the orbital period is slightly longer than the cut-off period, and

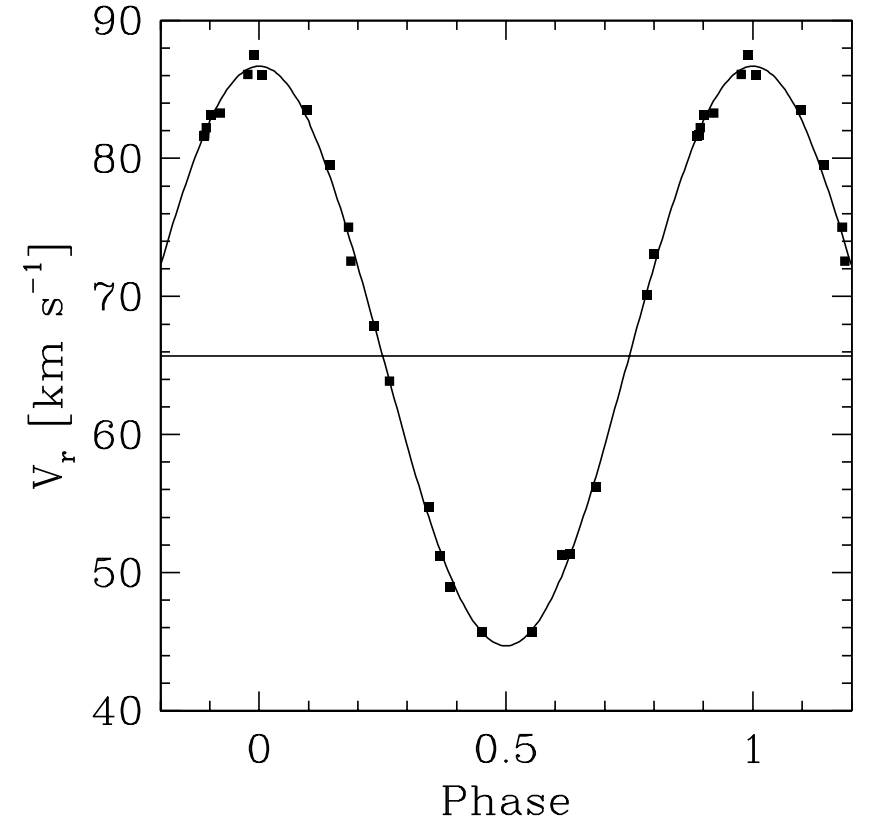

Fig. 1. Radial-velocity curve for C44.

the orbit appears to be slightly eccentric. The $\sigma(\mathrm{O}-\mathrm{C})$ of the residuals increases if the eccentricity is set to zero.

The frequency of definite spectroscopic binaries in NGC 1817 is $25.6 \%$ (10/39), and is close to the overall mean frequency of $23 \%$ obtained for red giants in open clusters by Mermilliod \& Mayor (1992). Five of the 10 definite binaries are located in the very centre, i.e. within a circle of $5^{\prime}$ in radius, centered on star $\mathrm{C} 13$. The projected binary distribution will be discussed below.

\subsection{Cluster velocity}

The mean cluster velocity is $+65.33 \pm 0.09(0.52$ s.e. $) \mathrm{km} \mathrm{s}^{-1}$ from 32 members, excluding the spectroscopic binaries without orbits. This is the first accurate determination of the mean velocity of NGC 1817. Previously, only one radial-velocity measurement with an error of $7 \mathrm{~km} \mathrm{~s}^{-1}$ for each of 5 stars was available (Friel et al. 1989).

\section{Colour-magnitude diagram}

Figure 4 presents the colour-magnitude diagramme for all 88 candidate red giants observed. The radial-velocity members are plotted as filled circles, the binary members as open circles, and the radial-velocity non-members as small dots. The curve overplotted is the isochrone from Girardi et al. (2000) for $\log t=8.90$ and $z=0.008$. The subsequent discussion will be based only on the radial-velocity members.

The main feature of the colour-magnitude diagram is the vertical clump extending over nearly 1 mag. with small scatter around $B-V=1.10$. The large vertical extension of the clump is well reproduced by the theoretical isochrone of Girardi et al. (2000) which matches simultaneously the separation between the first and second ascending giant branch and the extension of the clump. We conclude that no anomaly is present at this age. 
Table 4. Orbital elements of four spectroscopic binaries.

\begin{tabular}{lcccc}
\hline \hline Element & $\mathrm{C} 44$ & $\mathrm{C} 56$ & $\mathrm{C} 164$ & 1081 \\
\hline$P[\mathrm{~d}]$ & 68.0293 & 1262.9 & 165.760 & 4.30864 \\
& .0058 & 7.9 & .021 & .00025 \\
$T[\mathrm{HJD}-2400000]$ & 49979.24 & 48109. & 49771. & 52068.88 \\
& .11 & 20. & 10. & .85 \\
$e$ & 0.000 & 0.723 & .020 & 0.011 \\
& .010 & .018 & .007 & .033 \\
$\gamma\left[\mathrm{km} \mathrm{s}^{-1}\right]$ & 65.69 & 65.00 & 64.69 & 32.01 \\
& .16 & .12 & .14 & .74 \\
$\left.\omega{ }^{\circ}\right]$ & & 162.7 & 236. & 293. \\
& & 6.1 & 22. & 69. \\
$K\left[\mathrm{~km} \mathrm{~s}^{-1}\right]$ & 21.00 & 5.89 & 26.09 & 28.65 \\
& .22 & .41 & .20 & .50 \\
$f(m)\left[M_{\odot}\right]$ & 0.0654 & 0.0088 & 0.3054 & 0.0105 \\
& .0020 & .0026 & .0071 & .0005 \\
$a \sin i[\mathrm{Gm}]$ & 19.64 & 70.6 & 59.45 & 1.697 \\
& .20 & 7.3 & .46 & .030 \\
$\sigma(\mathrm{O}-\mathrm{C})\left[\mathrm{km} \mathrm{s}^{-1}\right]$ & 0.72 & 0.45 & 0.62 & 0.74 \\
$n_{\text {obs }}$ & 24 & 18 & 26 & 12 \\
\hline
\end{tabular}

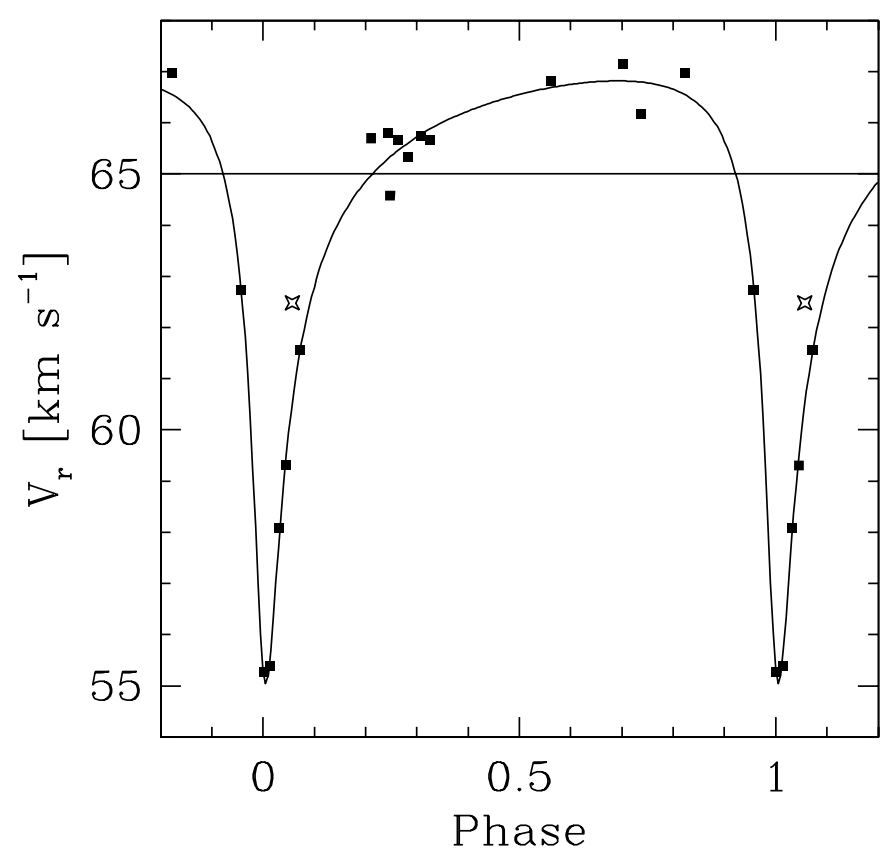

Fig. 2. Radial-velocity curve for C56.

The location of the spectroscopic binaries C44 and C164 can be explained by the combined light of a red-giant primary and a hotter secondary. The brightest binary, C56, lies to the right of the isochrone. Other binary members in a similar position have been found in two other clusters, NGC 6940 (Mermilliod \& Mayor 1989) and NGC 2360 (Mermilliod \& Mayor 1990). Two stars, C212 and \#1117 (Tian 117), occupy positions on the colour-magnitude diagram that are clearly inconsistent with the main cluster locus. Star \#1117 has position in the colour-magnitude diagram a similar to that of stars S1063 and S1113 studied by van den Berg et al. (1999). $P\left(\chi^{2}\right)$ is small (0.066) and further observations may prove it is a binary. If it is not, its membership would be seriously questioned. A star

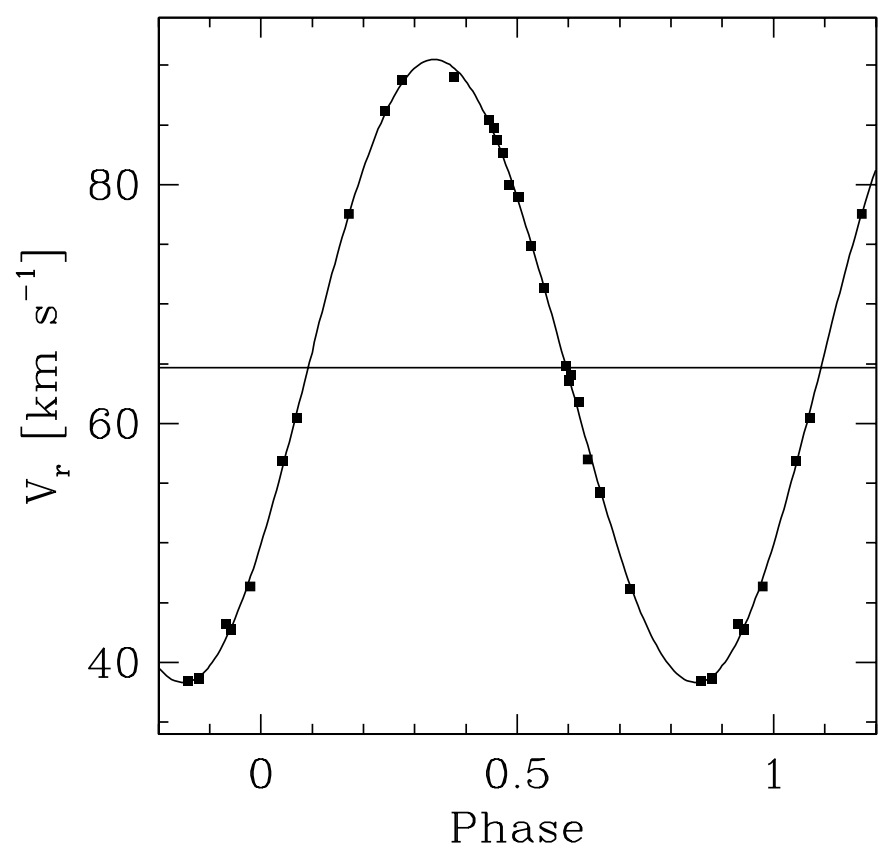

Fig. 3. Radial-velocity curve for C164.

located in an abnormal position in a colour-magnitude diagram should be considered as a cluster member only if it is binary and the systemic velocity is close to the cluster mean velocity. Photometry of star \#212 should also be confirmed.

\section{Mass-segregation}

The radial distribution of the definite spectroscopic binaries with a red-giant primary shows a strong concentration toward the cluster centre relative to the single red giants (Fig. 6), in contradiction to what Raboud \& Mermilliod (1994) found for intermediate-age clusters of similar ages included in their group $2(\log t=8.95)$. Mass-segregation in NGC 1817 is fully 


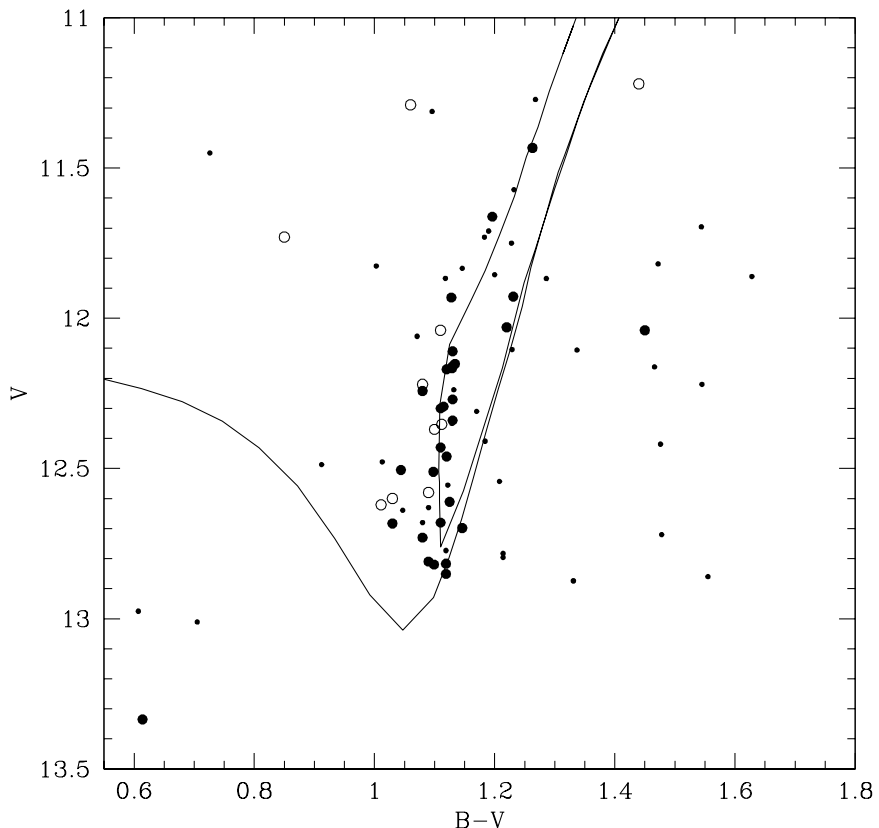

Fig. 4. Colour-magnitude diagram for the observed stars. Symbols distinguish the radial-velocity members (filled circles), the binary members (open circles), and the radial-velocity non-members (small dots). The curve is the isochrone from Girardi et al. (2000) for $\log t=8.90$ and $z=0.008$.

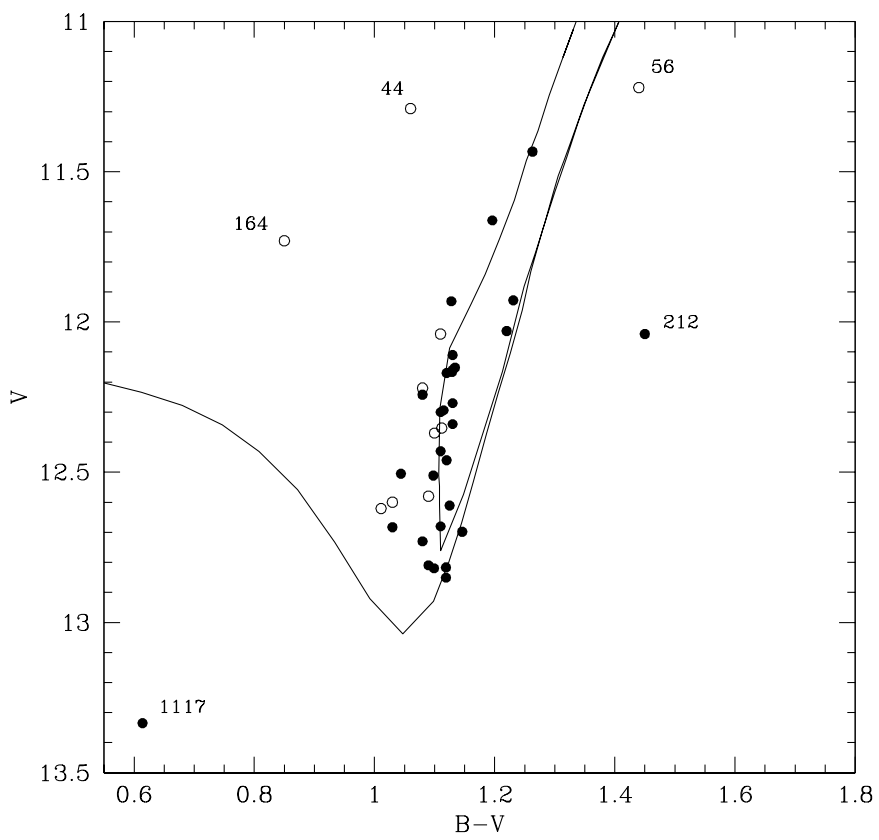

Fig. 5. Colour-magnitude diagram for the red-giant radial-velocity members. Binaries are indicated by open circles. The isochrone is the same as in Fig. 4.

developed and is nearly as pronounced as in the old open cluster M 67 (Mathieu \& Latham 1986). The inclusion or not of the probable binary (\#1456) or of stars \#1297 or \#1502 does not change this conclusion.

Indeed, Raboud \& Mermilliod (1994) have shown that mass segregation is not present between single and binary red giants in open clusters older than 0.6 Gyr, except for M 67 where it is strikingly evident. They found a hint, although not statistically significant, of an increase of the mass segregation with increasing cluster ages. NGC 1817 does not fit at all in this picture. With an age of $\log t=8.95$ it shows a mass segregation of the spectroscopic binaries as strong as that seen in M 67, in complete contradiction with the expectations of Raboud \& Mermilliod (1994).

In most clusters studied, we can safely assume that the surveys are quite complete, at least in the areas containing red giants. Therefore the observed differences between the other clusters of similar ages are not due to the incompleteness of the stellar samples, but rather are real. We might speculate that the lack of binaries in the central parts of the other clusters is due to the disruption of short-periods binaries, having reached contact during the evolution of the primary, and that this result is due to differences in the initial-period distributions. If there is an excess of periods shorter than 40 days, all of them will fill their Roche lobe and reach contact during evolution. Thus many binaries will be lost, decreasing the number of red giants and of binaries. But it would be a strange coincidence if this occured at the same time in all clusters. NGC 1817 shows that there is no simple relation between mass-segregation and age, nor with the number of red giant members. The main point is that the binary frequency in the central part of NGC 1817 is higher than the mean frequency in other open clusters with similar ages. It remains to be shown whether these clusters have different structural parameters (core radii, central densities), which could influence the dynamical evolution or even result from it. This is not so easy to do for these clusters, due to the small number of stars involved in comparison with globular clusters.

\section{Conclusions}

We have used radial velocities to confirm the membership of 21 red-giants in the central part of NGC 1817. New radialvelocity and $U B V R$ photoelectric observations have permitted us to identify 18 new red-giant members in the outer regions of NGC 1817 , out to a distance of $25^{\prime}$ from the centre. The radius of this cluster is at least twice as large as that previously tabulated $\left(10^{\prime}\right)$.

We have discovered ten definite spectroscopic binaries among the members and have determined three orbits. Three additional stars in the outer region of the cluster are possible spectroscopic-binary members. We have found a clear masssegregation between the single and binary red giant members, in the sense that binaries are more concentrated toward the cluster centre than single stars. In contrast with the results of Raboud \& Mermilliod (1994), the segregation in NGC 1817 is strikingly more pronounced than observed in other clusters of similar ages, in their group 2.

The red giant pattern in the colour-magnitude diagram of NGC 1817 has a large vertical range, which is well reproduced by the models of Girardi et al. (2000).

The case of NGC 1817 emphasizes that it is very important to cover the entire cluster area. Observations of the central part are not enough to describe completely the cluster properties.

Acknowledgements. The referee's comments on an earlier version of this paper encouraged us to obtain new observations, which confirmed and strengthened the earlier results. We are grateful to Dr. Leo Girardi 


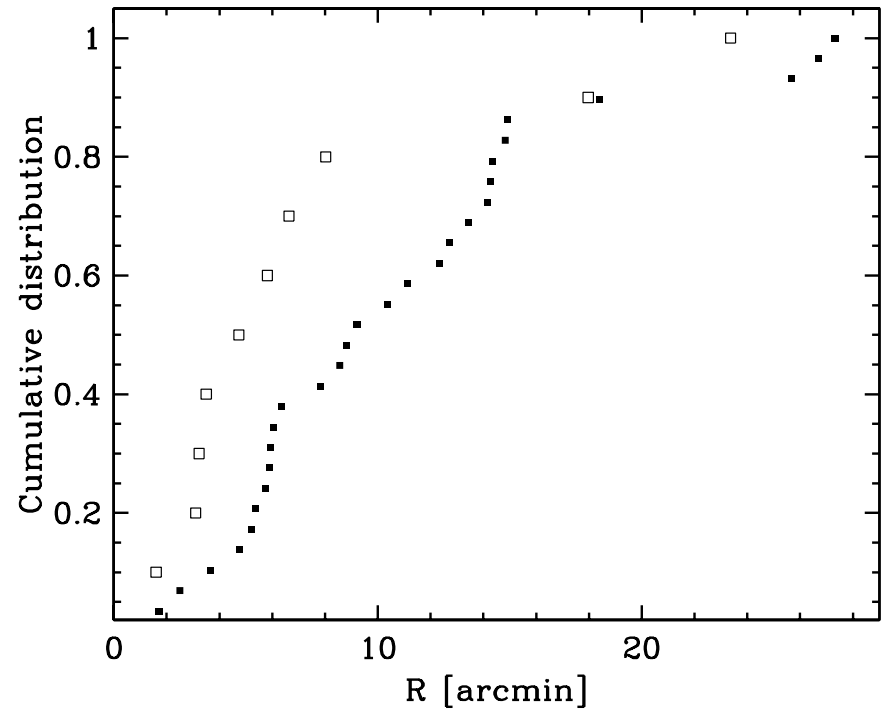

Fig. 6. Cumulative distributions of the single stars (filled squares) and definite spectroscopic binaries (open squares). The concentration of binaries towards the cluster centre is well marked.

for providing a copy of his evolutionary tracks for $Z=0.008$ and to Leonid Berdnikov for checking the photometry of 2 stars. DJJ was partially funded by a Royal Society European Science Exchange programme during the course of this project. We thank the staff and Director of the Isaac Newton Group of telescope operation on behalf of the British PPARC and Dutch NWO Science organisations. JCM acknowledges a research grant from the Swiss National Funds (FNRS).

\section{References}

Balaguer-Núñez, L., Tian, K. P., \& Zhao, J. L. 1998, A\&AS, 133, 387 Baranne, A., Mayor, M., \& Poncet, J.-L. 1979, Vistas Astron., 23, 279
Baranne, A., Queloz, D., Mayor, M., et al. 1996, A\&AS, 119, 373

Cuffey, J. 1938, Harvard Coll. Obs. Ann., 106, 39

Friel, E. D., \& Janes, K. A. 1993, A\&A, 267, 75

Friel, E. D., Liu, T., \& Janes, K. A. 1989, PASP, 101, 1105

Girardi, L., Bressan, A., Bertelli, G., \& Chiosi, C. 2000, A\&AS, 141, 371

Girardi, L., Mermilliod, J.-C., \& Carraro, G. 2000, A\&A, 354, 892

Harris, G. L. H., \& Harris, W. E. 1977, AJ, 82, 612

Landolt, A. U. 1992, AJ, 104, 340

Latham, D. W. 1992, in Complementary Approaches to Binary and Multiple Star Research, IAU Colloq. 135, ed. H. McAlister, \& W. Hartkopf, ASPC 32, 1000

Mathieu, R. D., \& Latham, D. W. 1986, AJ, 92, 1364

Mermilliod, J.-C., Clariá, J. J., Andersen, J., Piatti, A. E., \& Mayor, M. 2001, A\&A, 375, 30

Mermilliod, J.-C., \& Mayor, M. 1989, A\&A, 219, 125

Mermilliod, J.-C., \& Mayor, M. 1990, A\&A, 237, 61

Mermilliod, J.-C., \& Mayor, M. 1992, in Binaries as Tracers of Stellar Formation, ed. A. Duquennoy, \& M. Mayor (Cambridge Univ. Press), p. 183

Mermilliod, J.-C., \& Mayor, M. 1996, in Cool Stars, Stellar Systems, and the Sun, 9th Cambridge Workshop, ed. R. Pallavicini, \& A. K. Dupree, ASP Conf. Ser., 109, 373

Meynet, G., \& Maeder, A. 2000, A\&A, 361, 101

Purgathofer, A. 1964, Ann. Univ. Sternw. Wien 26 no 2

Raboud, D., \& Mermilliod, J.-C. 1994, A\&A, 289, 121

Raboud, D., \& Mermilliod, J.-C. 1998, A\&A, 333, 897

Stefanik, R. P., Latham, D. W., \& Torres, G., in Precise Stellar Radial Velocities, IAU Colloq. 170, ed. J. B. Hearnshaw, \& C. D. Scarfe, ASP Conf. Ser., 185, 354

Tian, K.-p., Ying, M.-g., Jin, J.-y., \& Xu, Z.-g. 1983, Ann. Shanghai Obs., 5, 227

Udry, S., Mayor, M., \& Queloz, D. 1999, in Precise Stellar Radial Velocities, IAU Colloq. 170, ed. J. B. Hearnshaw, \& C. D. Scarfe, ASP Conf. Ser., 185, 367

van den Berg, M., Verbunt, F., \& Mathieu, R. D. 1999, A\&A, 347, 866 